\title{
Pulmonary hypertension and HF with preserved EF
}

A population-based study has shown that pulmonary hypertension is common in patients with heart failure with preserved ejection fraction (HFpEF), and has indicated that both pulmonary venous hypertension and pulmonary arterial hypertension contribute to this condition.

Lam and colleagues diagnosed $8 \%$ of the 719 patients with a history of hypertension but no HF, and $83 \%$ of the 244 patients with HFpEF, as having pulmonary hypertension (pulmonary artery systolic pressure $[\mathrm{PASP}]>35 \mathrm{mmHg}$ ). The median PASPs for these patients were $28 \mathrm{mmHg}$ and $48 \mathrm{mmHg}$, respectively. PASP distinguished patients with HFpEF from hypertensive controls better than other echocardiographic measures and, according to Carolyn Lam, this observation raises "the exciting potential of using PASP to aid in the diagnosis of patients with HFpEF”. PASP remained considerably higher in the HFpEF group after adjusting for pulmonary capillary wedge pressure, suggesting that both pulmonary venous hypertension and pulmonary arterial hypertension contribute to pulmonary hypertension in these patients. PASP and the presence of pulmonary hypertension correlated with risk of mortality in the patients with HFpEF (median follow-up 2.8 years), which implicates an important role for pulmonary hypertension in the pathophysiology of HFpEF.

"Pulmonary hypertension may therefore represent a therapeutic target in patients with HFpEF," commented Lam, "lending support for trials of therapies such as phosphodiesterase-5 inhibitors", which includes the ongoing RELAX trial.

Bryony M. Mearns

Original article Lam, C. S. P. et al. Pulmonary hypertension in heart failure with preserved ejection fraction: a communitybased study. J. Am. Coll. Cardiol. 53, 1119-1126 (2009). 